\title{
"Multi Molecule Theory" with Gravitation as Binding Force between Molecules Can Explain Brownian Motion
}

\section{Satyavarapu Naga Parameswara Gupta*}

Retired Assistant General Manager, Bhilai Steel Plant, Bhilai, Chhattisgarh, India

*Corresponding Author: Satyavarapu Naga Parameswara Gupta, Retired Assistant

General Manager, Bhilai Steel Plant, Bhilai, Chhattisgarh, India.
Received: August 17, 2020

Published: October 07, 2020

(C) All rights are reserved by Satyavarapu

Naga Parameswara Gupta.

\section{Abstract}

Here in this paper we try to explain one of the foundations of Nanobiotechnology, i.e., the Physics portion. This paper is based on FINAL results of $4^{\text {th }}$ paper we developed for explaining.

What are the foundational reasons of Brownian motion and how it works? The Physics and the calculations behind the force and individual velocities of molecules with relevant theoretical analysis as proposed in the FIRST paper. In the fourth paper, more than 7000 iterations were conducted, and concluded 'Gravitation' is the final force of attraction between molecules. So, for explaining Brownian Motions, we can safely infer that the force field between Molecular pairs is Gravitation. In this paper we will discuss the positive theory behind MMT.

We also observed that "Gravitation" as the force of attraction is NOT sufficient to give enough force between a single pair of Molecules. But the collective vectoral sum of these forces of Multiple molecules in MMT, with sufficient number of iterations will explain these motions. For this theory of MMT EXCEL simulations were developed about month back.

Keywords: Nanobiotechnology; Multi Molecule Theory; Dynamic Universe Model; Gravitation; Force Field; Subbarao Simulations; Grazing Radiation Frequency Changes; Formation of Elements; Nucleosynthesis

\section{Introduction}

Recently a new branch of science using Cell Biology, Bio-Chemistry and Particle Physics called Nanobiotechnology. Here in this paper, we explain the foundational reasons of Brownian Motion by the way of Multi-Molecule theory using the concepts of Dynamic Universe Model. We will try to modify SITA simulation software for using in this platform and we call this as Subbarao Simulations.

\section{Brownian motion}

The age old Brownian motion is observed by anyone in Liquids, gases easily, and in solids with high end electron microscopes. In liquids and gasses, the key roles are done by the sizes of the particles. The colloids of liquids are good examples for this motion for $1 \mathrm{~nm}$ in diameter Particles. This diameter corresponds to the size of single molecules, biomolecules such as DNA, RNA, proteins. See the very good descriptions in the paper 'Life at low Reynolds number' by Purcell [1]. Now in this paper we will try to develop some equations using molecular forces, Brownian motions, coefficient of diffusion etc., using this Multi Molecule Theory instead of the age old 'single Molecule theory'.

\section{History}

In 1959, Alder and Wainwright [5] used an IBM 704 computer to simulate perfectly elastic collisions between hard spheres. They said "this many-body problem" was solved numerically using simultaneous equations of motion. Numerical methods give raise to errors always. This method solves many problems in both equilibrium and nonequilibrium statistical mechanics [5]. Probably the first simulation of matter, Gibson., et al. simulated radiation damage of solid copper by using a Born-Mayer type of repulsive interac- 
tion along with a cohesive surface force. In his paper " Correlations in the Motion of Atoms in Liquid Argon" in 1964, Rehman A., said "The pair-correlation function and the constant of self-diffusion are found to agree well with experiment; the latter is $15 \%$ lower than the experimental value. The spectrum of the velocity autocorrelation function shows a broad maximum in the frequency region $\omega=0.25(\mathrm{kBT} / \hbar)$ ". He used a system of 864 particles interacting with a Lennard-Jones potential and obeying classical equations of motion [6]. These are some of the N-body problem type solutions about 60 years back.

\section{Mathematical background for multi molecule theory of nano- biotechnology}

Let us assume an inhomogeneous set of N Molecules as a Colloid. This colloid is mix of water molecules, Individual proteins, polymers of living matter such as DNA, RNA, actin or microtubules, molecular motors etc. Water is universal solvent, universally available, so we take water + colloid particles for this theory. These don't react with each other chemically. All these molecules behave like bouncing rubber balls, sizes are different All these set will have different masses in accordance with their type. This colloid will lead to have Brownian motions of the NanoBio-Particles due to mutual gravitation attraction forces between individual particles. We will consider the gravitation influence of Earth, Sun, Moon etc., also on this colloid. Lets take total number of molecules to be 133 , to use modified SITA calculations. We call these calculations as SUBBARAO Simulations or Multi Molecule Theory (Later we will call as 'SSMMT'). For a broader perspective, let us call this set of all the systems of point masses as an Ensemble. Let us further assume that there are many Ensembles each consisting of a different number of systems with different number of point masses. Similarly, let us further call a group of Ensembles as Aggregate. Let us further define a Conglomeration as a set of Aggregates and let a further higher system have a number of conglomerations and so on and so forth.

We will start with 133 particles/molecules in a Micro-cubical cubical in a glass beaker. We will assume a Micro-Cubicle in this beaker with invisible elastic walls. We will use 3D Cartesian coordinates with some appropriate center of its axes in this beaker.

All the boundaries are perfectly elastic. Any Nanobio-Particle which hits the boundary will return according to Newtons laws. We will take nano distances and pico-second times as appropriate. To this case. We assume all the molecules to be perfect elastic spheres. There will be bumping and collisions between particles, and each pair will move and trace their path back after the elastic collision between the two. We can detect collisions by SSMMT software by two bumping particles when the center to center distance is less than or equal to the sum of two molecular radii of these Biospheres.

So, it is obvious that the distance between the two molecules will increase and their velocities reverses after each pair's collision.

In this paper we will not consider gravitational repulsion at very low distances, only the bumping will happen at that distance, later we will introduce this concept in a next paper....

Similarly, we will introduce the Viscosity forces in another forthcoming paper.

Initially, let us assume a set of $\mathrm{N}$ mutually gravitating point masses in a system under Newtonian Gravitation. All these Nanobio-particles will have some finite radii ri which we will use for the calculation of bumping or collision in the SSMMT software.

Let the $\mathrm{a}^{\text {th }} \wedge$ point mass has mass $\mathrm{m}_{\alpha}$ and is in position $\mathrm{x}_{\alpha}$. In addition to the mutual gravitational force, there exists an external $\mathrm{f}_{\text {ext }}$, due to other systems, ensembles, aggregates, and conglomerations etc. which also influence the total force $\mathrm{F}_{\alpha}$ acting on the point mass $_{\alpha}$. In this case, the $\mathrm{f}_{\text {ext }}$ is not a constant universal Gravitational field but it is the total vectorial sum of fields at $x_{\alpha}$ due to all the external to its system bodies and with that configuration at that moment of time, external to its system of $\mathrm{N}$ point masses.

Total Mass of system $=M=\sum_{\alpha=1}^{N} m_{\alpha}$

Total force on the point mass ${ }_{\alpha}$ is $\mathrm{F}_{\alpha^{\prime}}$ Let $\mathrm{F}_{\mathrm{a} \beta}$ is the gravitational force on the $\mathrm{a}^{\text {th }}$ point mass due to $\mathrm{b}^{\text {th }}$ point mass.

$$
F_{\alpha}=\sum_{\substack{\alpha=1 \\ \alpha \neq \beta}}^{N} F_{\beta}-m_{\alpha} \nabla_{\alpha} \Phi_{e x t}(\alpha)
$$

Moment of inertia tensor (We will assume moment inertia is the dominating, but for theoretical calculations for this paper we will ignore Viscus forces).

Total aggregate equations: (Aggregate consists of many ensembles and systems)

Assuming these forces are conservative, we can find the resultant force by adding separate forces vectorially from equations (1) and (2): 


$$
\Phi_{e x t}(\alpha)=-\sum_{\substack{\beta=1 \\ \alpha \neq \beta}}^{N^{\gamma}} \frac{G m_{\beta}^{\gamma}}{\left|x^{\gamma / \beta}-x^{\gamma \alpha}\right|}-\sum_{\substack{\beta=1 \\ \alpha \neq \beta}}^{N^{\gamma /}} \frac{G m_{\beta}^{\sigma \gamma}}{\left|x^{\delta / \beta}-x^{\delta / \alpha}\right|}
$$

The full development of all equations up to equation 3 are the basis of Dynamic Universe model and are available in many published papers. This paper shows how to apply these equations of Dynamic Universe Model to Multi Molecule theory (MMT).

The Equation 3 is the main powerful equation, which gives many results that are not possible otherwise today. This tensor can be subdivided into 21000 small equations without any differential equations or integral equations. Hence, this way of calculations provides a solution of X, Y, Z values of coordinates, velocities and accelerations of each Molecule in the setup for that particular instant of time. It uses a different type of mathematics based on Newtonian physics. This mathematics used here is relatively simple and straightforward. For all the mathematics, and the Excel based software, details are explained in the three books published by the author [7-9].

A point to be noted here is that the Dynamic Universe Model never reduces to General relativity on any condition. It uses a different type of mathematics based on Newtonian physics. This mathematics used here is simple and straightforward. As there are no differential equations present in Dynamic Universe Model, the set of equations give single solution in $\mathrm{x} y \mathrm{z}$ Cartesian coordinates for every point mass for every time step. All the mathematics and the Excel based software details are explained in the three books published by the author [7-9]. In the first book, the solution to Nbody problem-called Dynamic Universe Model (SITA) is presented; which is singularity-free, inter-body collision free and dynamically stable. The Basic Theory of Dynamic Universe Model published in 2010 [7]. The second book in the series describes the SITA software in EXCEL emphasizing the singularity free portions. This book written in 2011 [8] explains more than 21,000 different equations. The third book describes the SITA software in EXCEL in the accompanying CD/DVD emphasizing mainly HANDS ON usage of a simplified version in an easy way. The third book is a simplified version and contains explanation for 3000 equations instead of earlier 21000 and this book also was written in 2011 [9]. Some of the other papers published by the author are available at refs [3,5,10-12].

This Subbarao Software can be used in many places like presently unsolved applications of MMT, and we will discuss in our future papers. Here we have shown that this 'SSMMT using Gravita- tion between molecules' approach can be used positively in Nano Biology Technology.

\section{Discussion and Conclusion}

This paper confirms the entry of Dynamic Universe Model in particle physics with its similarly developed Multi-Molecule Theory in the new subject of nanobiotechnology, which is high potential science and is a conglomeration of four main branches of science viz, Physics, Biology, and chemistry and Engineering.

Till now this Nanobiotechnology used Single molecule theory and all the experiments in the Laboratory were conducted on the basis of the SMT only. This SSMMT opens a window to conducting lab experiments in a more elaborate broader and wider way. We strongly believe that cheaper and simpler Lab equipment also will be available soon. There are many problems and difficulties for isolating a single molecule for observations via an optical microscope. With SSMMT such problems will be reduced. Basic advantage being, we need not to Isolate UPTO a single molecule level.... Lets hope for the best.

This branch of science is very powerful and versatile.

A humble attempt is made to develop mathematical background for the Subbarao Simulation of Multi Molecule theory for an oral presentation in the upcoming conference Nanobiotech 2020.

\section{Acknowledgements}

I thank Maa Vak for giving me continuous Guidance on this new work. I also thank Prof Caroline Kelly, Program Director, Nanobiotech 2020, for asking me to deliver a lecture on nanobiotechnology, which is a perfectly new subject for me. And she inspired me to do this work for the upcoming online Conference during August 3-4, 2020.

\section{Bibliography}

1. Purcell C. "Life at low Reynolds number". American Journal of Physics 45 (1977): 3-11.

2. Daniel Riveline. "Single molecule': theory and experiments, an introduction From Nanophysics for Health". Journal of Nanobiotechnology 11.1 (2013): S1.

3. Papai G., et al. "New insights into the function of transcription factor TFIID from recent structural studies". Current Opinion in Genetics and Development 21.2 (2011): 219-224.

4. Meglio A., et al. "Single DNA/protein studies with magnetic traps". Current Opinion in Structural Biology 19.5 (2009): 615622. 
5. Alder BJ and Wainwright TE. "Studies in Molecular Dynamics. I. General Method". The Journal of Chemical Physics 31.2 (1959): 459-466.

6. Rahman A. "Correlations in the Motion of Atoms in Liquid Argon”. Physical Review 136.2 (1964): A405-A411.

7. SNP Gupta. "Dynamic Universe Model: A singularity-free Nbody problem solution". VDM Publications, Saarbrucken, Germany (2010).

8. SNP Gupta. "Dynamic Universe Model: SITA singularity free software”. VDM Publications, Saarbrucken, Germany (2011).

9. SNP Gupta. "Dynamic Universe Model: SITA software simplified”. VDM Publications, Saarbrucken, Germany (2011).

10. SNP Gupta. "Dynamic Universe Model Predicts the Live Trajectory of New Horizons Satellite Going To Pluto". Applied Physics Research 7.4 (2015): 63-77.

11. JV Narlikar. "Introduction to cosmology". Foundation books, New Delhi, India (1983).

12. SNP Gupta. "Dynamic Universe Model Explains the Variations of Gravitational Deflection Observations of Very-LongBaseline Interferometry". Applied Physics Research 6.4 (2014): 1-16.

\section{Assets from publication with us}

- Prompt Acknowledgement after receiving the article

- Thorough Double blinded peer review

- Rapid Publication

- Issue of Publication Certificate

- High visibility of your Published work

Website: www.actascientific.com/

Submit Article: www.actascientific.com/submission.php Email us: editor@actascientific.com

Contact us: +919182824667 